\title{
Desfolha artificial simulando danos de pragas na cultura do girassol (Helianthus annuus L., Asteraceae)
}

\author{
Izidro dos Santos de Lima Junior ${ }^{1}$, Thiago Ferreira Bertoncello ${ }^{2}$, Elmo Pontes de Melo ${ }^{3}$, \\ Paulo Eduardo Degrande 4 , Cássio Kodama ${ }^{5}$
}

\section{RESUMO}

O girassol está sujeito às perdas de área foliar por diferentes fatores, dentre eles os insetos desfolhadores, contra aos quais geralmente são dirigidas aplicações de inseticidas na cultura. A desfolha artificial em plantas de importância econômica é uma metodologia útil na simulação de ataques dessas pragas em lavouras na determinação dos níveis de dano econômico. O objetivo deste estudo foi avaliar componentes de produção das plantas de girassol submetidas a níveis crescentes de desfolha de 0,10, 25, 50, 75 e 100\%, realizada em três distintos estádios fenológicos da cultura, a saber: V6 (seis folhas com no mínimo 4,0 cm de comprimento), R1 (quando a inflorescência circundada pela bráctea imatura torna-se visível) e R5.5 (50\% das flores do disco estão fertilizadas ou em antese), perfazendo um total de 18 tratamentos, os quais foram dispostos em blocos ao acaso, com quatro repetições. Para todos os componentes de produção avaliados (diâmetro do capítulo, biomassa total de sementes da planta e biomassa de 100 aquênios) houve efeito significativo da interação dos tratamentos, evidenciando que o efeito da desfolha será dependente do estágio fenológico da planta. O estádio R5.5 foi mais sensível à desfolha, ocasionando perdas em todos os componentes de produção avaliados.

Palavras-chave: Nível de dano, área foliar, Chlosyne lacinia saundersii, Helianthus annuus.

\section{ABSTRACT}

\section{Artificial defoliation simulating pest damage on sunflower}

Loss of leaf area in sunflower may be caused by different factors, among them by defoliating insects, which are generally controlled with insecticide applications. Artificial defoliation in plants of economic value is a useful methodology to simulate attacks of pests on crops aiming at determining levels of economic damage. The objective of this study was to evaluate production components of sunflower subjected to increasing levels of defoliation $0,10,25$, 50, 75 and 100\% in three different phenological crop stages: V6 (six leaves with minimum of $4.0 \mathrm{~cm}$ in length), R1 (when inflorescence surrounded by immature bracts becomes visible) and R5.5 (50\% of the disk flowers are fertilized or in anthesis). The experiment consisted of 18 treatments, in a randomized block design with four replicates. There was significant effect of treatment interaction for all components of production evaluated (head diameter, total weight of seeds per plant and weight of one hundred seeds), showing that the effect of defoliation depends on the phenological stage of the plant. The R5.5 stage was more sensitive to defoliation, causing losses in all evaluated production components.

Key words: Greenhouse, phenological stage, defoliation level, Chlosyne lacinia saundersii, Helianthus annuus.

Recebido para publicação em agosto de 2008 e aprovado em novembro de 2009

${ }^{1}$ Engenheiro agrônomo, Mestre. Faculdade de Ciências Agrárias (FCA). Universidade Federal da Grande Dourados, Rodovia Dourados-Ithaum, Km 12, Caixa Postal 533, 79804970 Dourados, MS, Brasil. izidrojunior@gmail.com

${ }^{2}$ Engenheiro agrônomo, Mestre. Faculdade de Ciências Agrárias (FCA). Universidade Federal da Grande Dourados, Rodovia Dourados-Ithaum, Km 12, Caixa Postal 533, 79804970 Dourados, MS, Brasil. thiagobertoncello@gmail.com

${ }^{3}$ Engenheiro agrônomo, Doutor. Faculdade de Ciências Agrárias (FCA). Universidade Federal da Grande Dourados, Rodovia Dourados-Ithaum, Km 12, Caixa Postal 533, 79804970 Dourados, MS, Brasil. epmeloagro@yahoo.com.br

${ }^{4}$ Engenheiro agrônomo, Doutor. Faculdade de Ciências Agrárias (FCA). Universidade Federal da Grande Dourados, Rodovia Dourados-Ithaum, Km 12, Caixa Postal 533, 79804970 Dourados, MS, Brasil. paulodegrande@ufgd.edu.br

${ }_{5}^{5}$ Engenheiro agrônomo, Faculdade de Ciências Agrárias (FCA). Universidade Federal da Grande Dourados, Rodovia Dourados-Ithaum, Km 12, Caixa Postal 533, 79804-970 Dourados, MS, Brasil. cássio_kodama@hotmail.com 


\section{INTRODUÇÃO}

O girassol (Helianthus annuus L., Asteraceae), planta originária da América do Norte, é cultivado em várias partes do mundo e apresenta cerca de 18 milhões de hectares plantados (Camargo \& Amabile, 2001).

Em condições de campo, o girassol está sujeito às perdas de área foliar por diferentes fatores, dentre eles insetos desfolhadores, cujo principal representante é a lagarta-do-girassol, Chlosyne lacinia saundersii Doubleday \& Hewitson, 1849 (Lepidoptera: Nymphalidae), seu ataque ocorre principalmente de outubro a maio (Gallo et al., 2002).

A desfolha artificial em plantas de importância econômica, como a soja e o feijão, é uma metodologia útil para simular danos ocorrentes em lavouras, como os frequentes ataques de pragas desfolhadoras ou eventual chuva de granizo (Fazolin \& Estrela, 2003). Essa metodologia permite mensurar seguramente quanto de desfolha a cultura pode suportar em determinado estádio fenológico, quantificando a perda de produtividade em diferentes níveis de desfolha.

A perda de produção ocasionada pela desfolha em milho está relacionada à intensidade da perda de área foliar e ao estádio de crescimento em que ele é submetido (Lauer et al., 2004). Muro et al. (2001) descrevem que as maiores reduções na produção do girassol ocorrem com 100\% da desfolha nos estádios reprodutivos, isso acontece porque as plantas estão mais sensíveis à desfolha extrema nesses estádios.

Dessa forma, as informações obtidas neste estudo, tal como a determinação do nível de dano econômico, podem reduzir os custos do controle químico, evitar ou retardar a seleção de populações resistentes, evoluídas a partir das aplicações contínuas de inseticidas (Fazolin \& Estrela, 2003), minimizar a contaminação do ambiente (solo, água, atmosfera e seres vivos) e diminuir danos acidentais pelo uso irracional dos produtos, já que o controle de desfolhadores será feito somente quando necessário.

Sendo assim, o objetivo deste estudo foi avaliar os componentes de produção das plantas de girassol submetidas a níveis crescentes de desfolha artificial em três estádios fenológicos da cultura.

\section{MATERIAL E MÉTODOS}

O experimento foi realizado em vasos, em condições de casa de vegetação, na Faculdade de Ciências Agrárias (FCA) da Universidade Federal da Grande Dourados (UFGD), em Dourados (22 `14' de Latitude Sul, $54^{\circ} 44^{\prime}$ ' de Longitude Oeste e Altitude de $452 \mathrm{~m}$ ), Estado de Mato Grosso do Sul, entre os meses de abril e agosto de 2005.

O solo utilizado no experimento foi oriundo de horizonte B de um Latossolo Vermelho distroférrico (Embrapa,
2006), coletado na FCA da UFGD. Os valores da caracterização química da amostra foram: 0,26 (K), 1,91 (Al), 1,4 (Ca), 0,1 (Mg), 11,1 ( $\mathrm{H}+\mathrm{Al}), 1,7$ (SB) e 12,86 (CTC), em cmol dm-3; 23,7 (M.O.), em mg dm ${ }^{-3} ; 4,9\left(\mathrm{pH} \mathrm{H}_{2} \mathrm{O}\right)$ e 4,2 (pH CaCl2); e física 80,42 (argila), 9,34 (silte), 10,23 (areia total) e 54,47 (VTP), em \%, e 1,07 Ds e 2,35 Dp, em g cm${ }^{-3}$, determinados de acordo com Embrapa (1997). Com os valores de densidade de partícula (Dp) e densidade aparente do solo (Ds) obteve-se o volume total de poros (VTP\%) utilizado no procedimento para controle da umidade nos vasos (ocupou-se com água 60\% do VTP do solo).

O conteúdo inicial de solo nos vasos foi de $8 \mathrm{~kg}$, o qual recebeu 3,75 $\mathrm{g} \mathrm{dm}^{3}$ de calcário dolomítico (dosagem determinada utilizando-se um V\% para a cultura do girassol de 70) incubado por um período de 21 dias. Após a incubação do corretivo, o solo foi seco ao ar, peneirado em malha de 2 $\mathrm{mm}$ de abertura para homogeneização da sua amostra, recebendo posteriormente a adubação recomendada (Tabela 1) para experimentos em casa de vegetação, de acordo com Novais (1991). Essa adubação foi aplicada, veiculada em 50 $\mathrm{mL}$ de água destilada com auxílio de pipeta, sobre o solo contido em cada vaso, espalhado sobre uma superfície de lona negra de polietileno. A adubação de macronutrientes primários e secundários foi separada da adubação para micronutrientes. Em seguida, esse conteúdo de solo contendo a adubação foi homogeneizado em saco plástico, retornando aos vasos de origem. A quantidade de solo remanescente nos vasos foi de $7,5 \mathrm{~kg}$.

Cada vaso recebeu sete sementes do cultivar de girassol M 734 tratadas com inseticida Thiamethoxam (Cruiser ${ }^{\circledR}$ ) e semeadas no dia 30/09/05 as quais emergiram em 05/10/ 05. Oito dias após, foi efetuado o desbaste, permanecendo apenas uma planta por vaso. Quando as plantas se encontravam com 40 dias (V2), foi feita aplicação do inseticida Thiamethoxam (Actara ${ }^{\circledR} 250 \mathrm{WG}$ ) na dose de $100 \mathrm{~g} \mathrm{ha}^{-1}$.

Foram estudados seis níveis de desfolha: 0, 10, 25, 50, 75 e 100\%, realizada nas épocas: V6 (seis folhas com no mínimo 4,0 cm de comprimento), R1 (a inflorescência circundada pela bráctea imatura torna-se visível) e R5.5 (50\%

Tabela 1. Recomendação utilizada no experimento conduzido em casa de vegetação

\begin{tabular}{lc}
\hline Nutrientes & mg dm$^{-3}$ de solo \\
\hline $\mathrm{N}$ & 150 \\
$\mathrm{P}$ & 80 \\
$\mathrm{~K}$ & 75 \\
$\mathrm{~S}$ & 905 \\
$\mathrm{~B}$ & 0,5 \\
$\mathrm{Cu}$ & 1,5 \\
$\mathrm{Zn}$ & 5,0 \\
$\mathrm{Mo}$ & 0,1 \\
$\mathrm{Fe}$ & 1,55 \\
$\mathrm{Mn}$ & 1,22 \\
\hline
\end{tabular}

Fonte: Adaptado de NOVAIS (1991) 
das flores do disco estão fertilizadas ou antese), de acordo com a escala fenológica proposta por Schneiter \& Miller (1981), perfazendo 18 tratamentos, os quais foram dispostos em blocos ao acaso com quatro repetições, cada vaso equivalia a uma repetição. As plantas eram desfolhadas apenas uma vez e eram conduzidas até a colheita.

Para obtenção dos níveis de desfolha, estabelecidos nos estádios fenológicos estudados, foram retirados, com auxílio de uma tesoura, de todas as folhas, os percentuais correspondentes ao desfolhamento estabelecido. A colheita para coleta de dados foi efetuada aos 104 dias após a emergência (17/01/2006). Os componentes de produção avaliados foram: diâmetro do capítulo, número de aquênios, biomassa total de aquênios de cada planta e biomassa de 100 aquênios. Somente os dados de números de aquênios foram transformados a raiz quadrada de $\mathrm{x}$ + 0,5 para a análise da variância e reconvertidos para apresentação neste trabalho. Os dados obtidos foram submetidos à análise de variância, utilizando-se o programa computacional SISVAR.

Os dados referentes aos níveis de desfolha foram submetidos à análise da variância da regressões linear e múltipla ( $\mathrm{P}<0,05)$.

Para as interações significativas $(\mathrm{P}<0,05)$, investigou-se a análise das regressões linear e múltipla entre os níveis de desfolha dentro de cada estádio.

\section{RESULTADOS E DISCUSSÃO}

A análise de variância para os componentes de produção avaliados - diâmetro de capítulo, número de sementes, massa total e massa de 100 aquênios - foi significativa ( $\mathrm{P}<0,05)$ para estádio fenológico, nível de desfolha e interação. O efeito significativo da interação encontrada entre os fatores estudados evidencia que a desfolha afeta a produção diferentemente nos estádios estudados.

Alguns estudos com girassol e feijão descrevem essa interação entre nível de desfolha e estádio de desenvolvimento, relacionando as maiores perdas quando a desfolha ocorre nos estádios reprodutivos (Moura, 1999; Lauer et al., 2004). Resultados semelhantes foram descritos com outras espécies, como relatado por Nurmberg et al. (1999), estudando desfolha artificial em batata (Solanum tuberosum L.), que o mesmo nível de desfolha pode ocasionar diferentes perdas, dependendo do estádio em que é efetuada.

A redução do diâmetro do capítulo em função do nível de desfolha indica que sua ocorrência no florescimento e enchimento de aquênios pode reduzir a produção, uma vez que o tamanho do capítulo, de acordo com Lakshmanrao et al. (1985), tem correlação significativa com o rendimento de grãos. Entre todos os componentes de produção avaliados, o diâmetro de capítulo teve o maior efeito sobre o rendimento de grãos (Figura 1).
A maior redução do tamanho de capítulo quando se desfolhou em 100\% a planta, no estádio R5.5, deve-se ao fato de a folha ser o principal aparato fotossintético, acumulando, além de nutrientes, compostos orgânicos que serão posteriormente translocados para os órgãos reprodutivos e os aquênios (Leite et al., 2005).

No estádio V6, o maior número de aquênios por planta ocorreu quando $50 \%$ da planta foi desfolhada, indicando que no estádio vegetativo o girassol suporta certo nível de desfolha, mas aquelas acima de 50\% podem levar à diminuição da produção. Fazolin \& Estrela (2003) atribuem a tolerância à desfolha, por serem as folhas remanescentes suficientes para a produção de fotoassimilados. Por outro lado nos estádios reprodutivos R1.1 e R5.5 houve diminuição linear no número de sementes com o aumento da desfolha (Figura 2).

Foi observado que a desfolha severa no estádio de enchimento de grãos pode ocasionar diminuição no número de aquênios e, consequentemente, na produção, pois, segundo Alvarez et al. (1992), o número de aquênios por capítulo está correlacionado positivamente com a produção.

Relacionando-se a biomassa de 100 aquênios com o nível de desfolha, observou-se que, para o estádio R5.5, o valor máximo foi observado com 50\% de desfolha, apresentando posterior redução. Para o estádio V6 foi verificado aumento de biomassa para desfolha de $100 \%$, fato que pode ser explicado pelo menor número de aquênios produzidos neste nível de desfolha. De acordo com Stone \& Pereira (1994), em estudos com arroz e feijão a redução em um ou mais componentes da produção pode, normalmente, levar ao incremento de outros, ou seja, a planta compensa a diminuição no número de grãos aumentando o seu peso (Figura 3). A maior biomassa de 100 aquênios no estádio V6 deve-se ao fato de que o estresse provocado pela desfolha ocorreu em um período inicial da cultura, o que garantiu tempo para recuperação das plantas, e elas ficaram com tamanho menor e folhas mais túrgidas, produzindo capítulos menores e com aquênios maiores.

Muitos insetos atacam a cultura do girassol no estádio de desenvolvimento V6, como Diabrotica speciosa (Germar) (Coleoptera: Crysomelidae) e Maecolaspis sp, (Gallo et al., 2002), podendo provocar a desfolha numa fase de pequena disponibilidade foliar e levar a planta à morte, dependendo das condições climáticas em que se encontram, sendo os danos mais severos causados decorrentes do consumo da área foliar pelos adultos. Em ensaio de laboratório, Hohmann \& Carvalho (1989) estimaram que o consumo foliar médio em feijão por $D$. speciosa foi de $0,70 \mathrm{~cm}^{2}$ por dia, podendo atingir 10,32 $\mathrm{cm}^{2}$ até o final do estádio adulto.

A biomassa total produzida por vaso seguiu o modelo linear na fase reprodutiva (R1.1 e R5.5) (Figura 4), evidenciando que após desfolha de $10 \%$ nestes estádios pode 


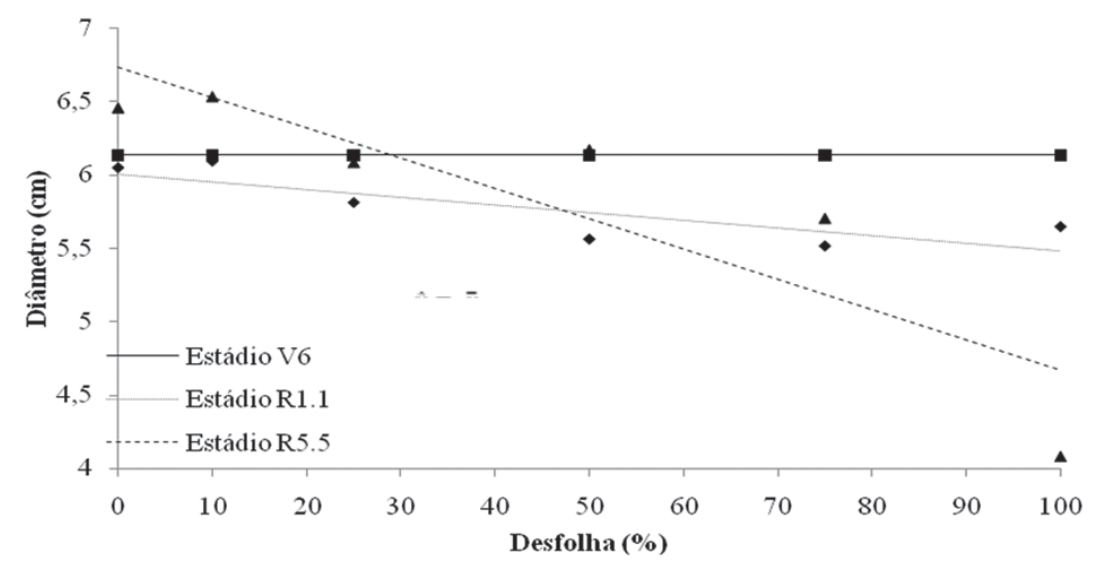

Figura 1. Diâmetro (cm) de capítulo nos diferentes estádios de desenvolvimento e níveis de desfolha do girassol.

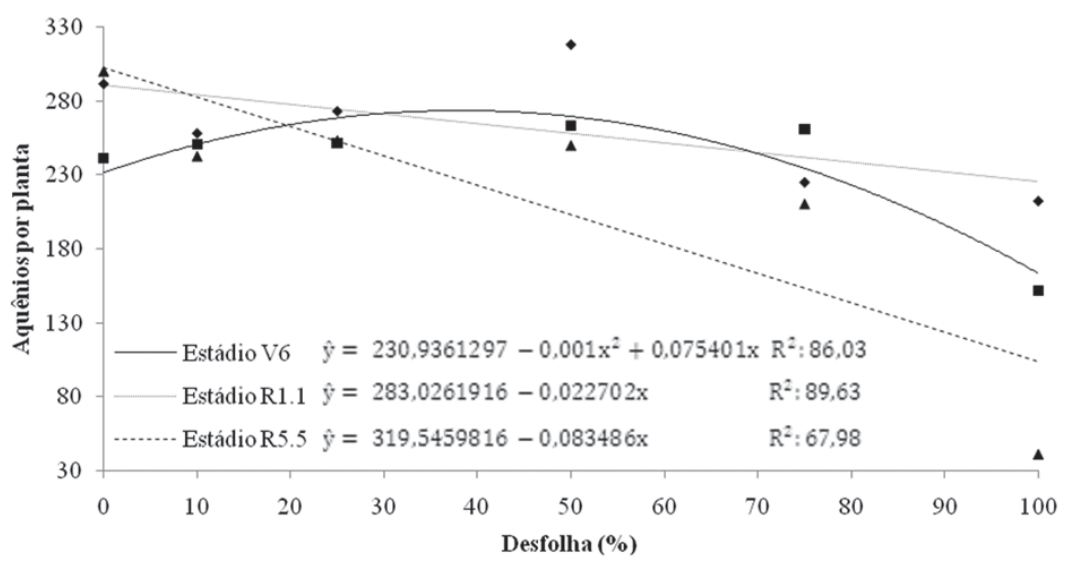

Figura 2. Número de aquênios nos diferentes estádios de desenvolvimento e níveis de desfolha do girassol.

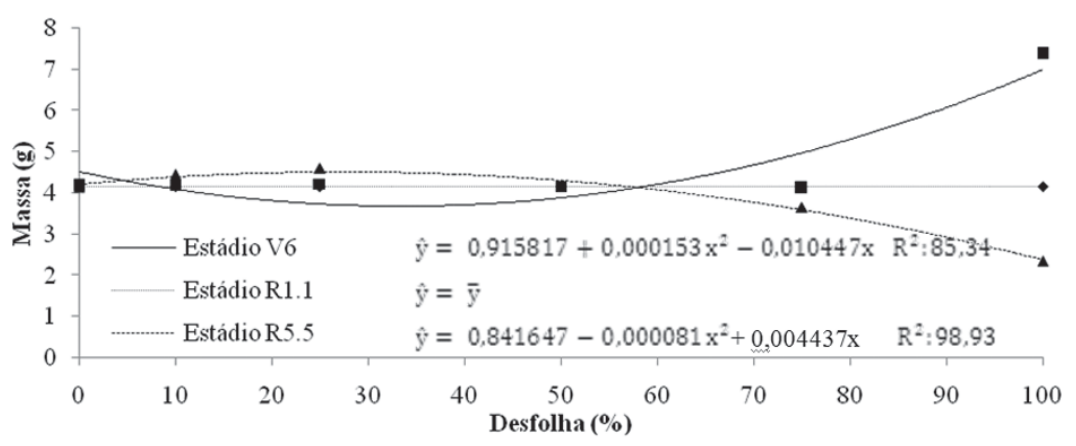

Figura 3. Massa de 100 aquênios nos diferentes estádios de desenvolvimento e níveis de desfolha do girassol.

ocorrer perda na produção. O modelo de regressão linear no estádio R1.1 demonstra que nesse período (florescimento) ocorre perda na produção pela desfolha, resultados que são corroborados pelos encontrados por Baydar \& Erbas (2007).

A regressão linear que se ajustou aos dados de produção mostra claramente que, na fase de enchimento de aquênios, qualquer desfolha pode acarretar em prejuízo. Steer et al. (1988) observara que a desfolha parcial ou total da planta durante o período da antese diminui a produção, o que, segundo Leite et al. (2005), pode ser atribuído ao fato de o rendimento de grãos depender amplamente da eficiência fotossintética da folha e da intensidade de transferência dos fotoassimilados para o grão na ocasião de sua formação e de seu enchimento. Isso demonstra que durante o estádio R5.5 ainda estava ocorrendo a translocação de fotoassimilados dos órgãos vegetais para estruturas reprodutivas. Por outro lado, Muro et al. (2001) constataram que desfolhas acima de $33 \%$ no estádio R.3 foram as que mais afetaram a produção do girassol. 


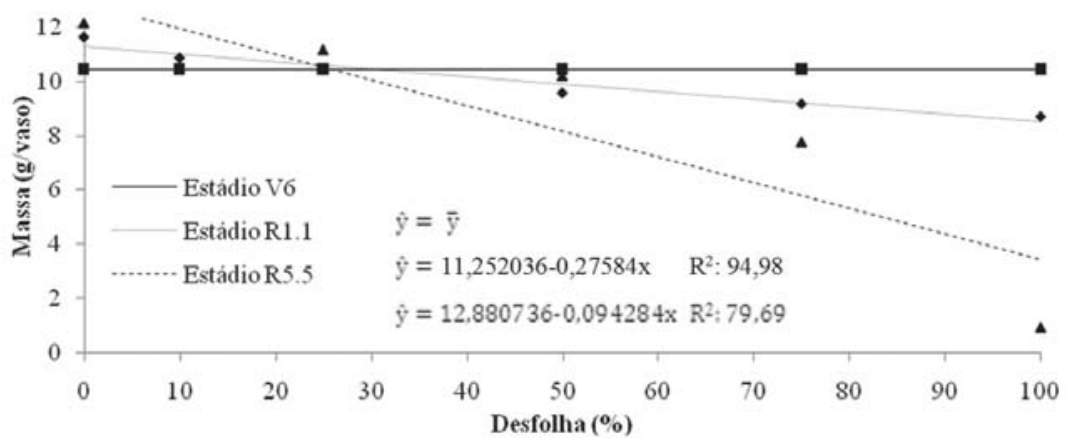

Figura 4. Massa total (g) nos diferentes estádios de desenvolvimento e níveis de desfolha do girassol.

Alguns trabalhos com desfolha artificial em diferentes culturas, como soja e feijão, demonstram que as maiores perdas na produção ocorrem no estádio de formação e enchimento de grãos (Diogo et al., 1997; Gazzoni \& Moscardi, 1998; Moura, 1999).

A desfolha no estádio R5.5 é ocasionada principalmente pela lagarta-do-girassol Chlosyne lacinia saundersii (Doubleday \& Hewitson, 1847) (Lepidoptera: Nymphalidae), que nos estádios iniciais de desenvolvimento mantém-se agregada, fazendo com isso uma desfolha severa em lugares localizados na lavoura. $\mathrm{O}$ ataque dessa praga entre os 50 e 70 dias de idade das plantas pode reduzir a produção em até $80 \%$ (Gallo et al., 2002). Então o conhecimento da desfolha suportada pelo girassol pode levar à diminuição na aplicação de inseticidas.

\section{CONCLUSÕES}

Os componentes de produção avaliados (diâmetro do capítulo, biomassa total de sementes de cada planta e biomassa de 100 aquênios) são afetados significativamente em função do nível de desfolha e, dependendo, do estágio fenológico em que a planta se encontra;

No estádio R5.5, o girassol foi mais sensível à desfolha, ocasionando perdas em todos os componentes de produção avaliados.

\section{REFERÊNCIAS BIBLIOGRÁFICAS}

Alvarez D, Luduena P \& Frutos E (1992) Correlation and causation among sunflower traits. In: $13^{\circ}$ International Sunflower Conference, Pisa. Proceeding, Paris: International Sunflower Association. p.957-962.

Baydar H \& Erbas S (2007) Defoliation effects on sunflower (Helianthus annus L.) seed yield and oil quality. Turk Journal Biology, 31:115-118.

Camargo AJA \& Amabile RF (2001) Identificação das principais pragas do girassol no Centro-Oeste. Brasília, Embrapa CPAC 4p. (Comunicado Técnico 50).

Diogo AM, Sediyama T, Rocha VS \& Sediyama CS (1997) Influência da remoção de folhas, em vários estádios de desenvolvimento, na produção de grãos e em outras características agronômicas da soja (Glycine max (L.) Merrill). Revista Ceres, 44:272-285.

Embrapa (2006) Sistema brasileiro de classificação de solos. $2^{\text {a }}$ ed. Rio de Janeiro, Cnps/Embrapa. 306p.
Embrapa (1997) Centro Nacional de Pesquisa de Solos. Manual de métodos de análise de solo. $2^{\text {a }}$ ed. Rio de Janeiro, Cnps/ Embrapa. 212p.

Fazolin M \& Estrela JLV (2003) Comportamento da cv. pérola (phaseolus vulgaris 1.) submetida a diferentes níveis de desfolha artificial. Ciência agrotecnologia, 27:978-984.

Gallo D, Nakano O, Neto SS, Carvalho RPL, Batista GC, Filho EB, Parra JRP, Zucchi RA, Alves SB, Vendramim JD, Marchini LC, Lopes JRS \& Omoto C (2002) Entomologia Agrícola. Piracicaba, FEALQ. 920p.

Gazzoni DL \& Moscardi F (1998) Effect of defoliation levels on recovery of leaf area, on yield and agronomic traits of soybeans. Pesquisa Agropecuária Brasileira, 33:411-424.

Hohmann CL \& Carvalho SM (1989) Pragas e seu controle. In: IAPAR (Ed.) O feijão no Paraná. Londrina, Instituto Agronômico do Paraná. p.217-246.

Lakshmanrao NG, Shambulingappa KG \& Kusumakumari P (1985) Studies on path-coeficiente analysis in sunflower. In: International Sunflower Conference, Mar del Plata. Proceedings, International Sunflower Association. p.733-735.

Lauer JG, Roth GW \& Bertram MG (2004) Impact of Defoliation on Corn Forage Yield. Agronomy Journal, 96:1459-1463.

Leite RMVBC, Brighenti AM \& Castro C (2005) Girassol no Brasil. $1^{\text {a }}$ ed. Londrina, Embrapa Soja. 641p.

Moura GM (1999) Efeito do desfolhamento no rendimento do feijoeiro. Pesquisa Agropecuária Brasileira, 34:57-62.

Muro J, Irigoyen I, Militino AF \& Lamsfus C (2001) Defoliation Effects on Sunflower Yield Reduction. Agronomy Journal, 93:634-637.

Novais RF, Neves JCL \& Barros NF (1991) Ensaio em ambiente controlado. In: Oliveira AJ, Garrido WE, Araujo JD, Lourenço S (Eds.) Métodos de pesquisa em fertilidade do solo. Brasília, EMBRAPA/SEA. 392p.

Nurmberg PL, Pinto CABP, Lambert ES \& Menezes CB (1999) Simulação de danos causados por insetos na planta de batata por meio de desfolhamento artificial. Ciência e Agrotecnologia, 23:468-472.

Schneiter AA \& Miller JF (1981) Description of sunflower growth stages. Crop Sciense, 21:901-903.

Steer BT, Hocking PJ \& Low A (1988) Dry matter, minerals and carbohydrates in the capitulum of sunflower (Helianthus annuus): Effects of competition between seeds, and defoliation. Field Crop Research,18:71-85.

Stone LF \& Pereira AL (1994) Sucessão arroz-feijão irrigados por aspersão. Efeitos de espaçamentos entre linhas, adubação e cultivar na produtividade e nutrição do feijoeiro. Pesquisa Agropecuária Brasileira, 29:521-533.

Rev. Ceres, Viçosa, v. 57, n.1, p. 023-027, jan/fev, 2010 